\title{
Physical Well-being
}

National Cancer Institute

\section{Source}

National Cancer Institute. Physical Well-being. NCI Thesaurus. Code C91779.

The general condition of one's body. 